\title{
Preface to the special issue on ambient noise seismology*
}

\author{
Xiaodong Song * \\ Department of Geology, University of Illinois at Urbana-Champaign, Urbana, IL 61801, USA
}

Not too long ago, seismic imaging of the Earth's interior relied almost exclusively on the illumination from energetic sources, such as earthquakes or artificial sources. However, recent theoretical and laboratory studies have shown that the impulse response (Green's function) of a structure between two receivers can be obtained from the cross-correlation of "random" noise wavefields recorded at the two receivers. The basic idea is that a random field contains coherent signals traveling between the receivers, which can be stacked and amplified while all other arrivals are canceled out in the cross-correlation. The idea has now found rapid applications in seismology. In particular, surface waves have been found to be most easily retrievable from the cross-correlations of the ambient seismic noise in the Earth.

The new area of research is expanding rapidly and has quickly become an important branch of seismological research. This field, which I use a general term ambient noise seismology here, is known as Green's function retrieval and is often called seismic interferometry in the exploration geophysics community (because of the cross-correlation involved). Major developments include new theories, new data processing techniques, imaging of Earth structures from ambient noise tomography, monitoring of subsurface processes, and characteristics of ambient noise sources.

The special issue contains 13 papers on this timely topic from researchers from China, France, Germany, and the U.S.. We hope that the collection can serve as a small window into this exciting field for the vast readership of the Chinese seismological community, while promoting scientific exchanges and communication between researchers in China and western countries. For readers who are interested in learning more about the

\footnotetext{
* Received 22 September 2010; accepted in revised form 23 September 2010; published 10 October 2010.

• Corresponding author. e-mail: xsong@illinois.edu

(c) The Seismological Society of China and Springer-Verlag Berlin Heidelberg 2010
}

subject, I list at the end of the preface a few review papers and books. The book edited by Wapenaar et al. (2008) contains a collection of 73 papers (including 22 papers published earlier in a supplement to the JulyAugust issue of Geophysics in 2006). The book by Schuster (2009) describes the theory and practice of seismic interferometry with an emphasis on applications in exploration seismology.

The papers in the special issue are largely organized by topics, including theory and methods, results of ambient noise tomography in various regions, studies of temporal changes of the media, and applications in planetary science. Below is a summary of each paper.

Weaver provides a pedagogic review of the concept of equipartition and its application to the retrieval of the Green's function from diffuse field. Snieder et al. review several formulations of equipartition and show that equipartitioning may not be sufficient for the Green's function retrieval. They also show how attenuation can be incorporated in Green's function retrieval of damped oscillating systems. Nowack shows that the crosscorrelation and integration over all sources in the construction of Green's function can be evaluated using Gaussian beams and demonstrates that the major contribution is from the sources near the stationary phase regions. Zhan and $\mathrm{Ni}$ report an error in an earlier analysis of Green's function retrieval and suggest that the inter-station distance may need to be large enough to obtain accurate Green's function from ambient noise correlation. Wang et al. present evidence for the emergence of $\mathrm{P}$ waves in noise cross-correlation of a small aperture array at high frequencies $(30-70 \mathrm{~Hz})$ and for the lack of surface waves, which are normally observed in cross-correlation at long periods.

Koper and Hawley investigate seismic noise at a single station and observe a rich variety of noise sources at different frequencies.

A number of contributions deal with seismic tomography in various regions using ambient noise, all of 
which use dispersion measurements of Green's functions to invert for 3D shear wave velocity structures of crust and upper mantle. Sun et al. study a large area of China and its surrounding regions using Rayleigh wave group and phase velocities from $8 \mathrm{~s}$ to $60 \mathrm{~s}$. Zheng et al. study northeastern Tibetan plateau and the adjacent region to its east (the Ordos block and the Sichuan basin) using Rayleigh wave phase velocities from $6 \mathrm{~s}$ to $50 \mathrm{~s}$. Fang et al. study North China using Rayleigh group velocities from $4 \mathrm{~s}$ to $30 \mathrm{~s}$, and Li et al. image crust and upper mantle of Italy using Love group velocities from $8 \mathrm{~s}$ to $34 \mathrm{~s}$.

Zhao et al. investigate temporal changes along the Parkfield section of the San Andreas fault and the surrounding area triggered by local, regional, and teleseismic earthquakes. Liu et al. compare different processing techniques for detecting temporal changes in the Earth.

Lastly, Sens-Schönfelder and Larose apply passive interferometry techniques to the Apollo 17 lunar seismic data, highlighting the potential of passive techniques for terrestrial and planetary seismology.

The special issue was put together in a very short time, which would not have been possible without the support of many people. I thank all the authors for their contributions and timely responses and all the reviewers for their reviews in a short time frame. Associate editor-in-chief Zhonghuai Xu was responsible for the article by Sun et al. as the editor of the special issue was a co-author of the paper. Finally, I'd like to thank the editorial staff of Earthquake Science for their effort and dedication in producing this high quality issue.

Following are some review papers and books on ambient noise seismology for reference.

Campillo M (2006). Phase and correlation in 'Random' seismic fields and the reconstruction of the Green function. Pure Appl Geophys 163(2-3): 475-502.

Gouédard P, Stehly L, Brenguier F, Campillo M, Verdière Y C, Larose E, Margerin L, Roux P, Sánchez-Sesma F J, Shapiro N $M$ and Weaver R L (2008). Cross-correlation of random fields: mathematical approach and applications. Geophys Prospecting 56: 375-393.

Larose E, Margerin L, Derode A, van Tiggelen B, Campillo M, Shapiro N M, Paul A, Stehly L and Tanter M (2006). Correlation of random wavefields: An interdisciplinary review. Geophysics 71(4): SI11-SI21.

Schuster G T (2009). Seismic Interferometry. Cambridge University Press, Cambridge, 272 pp.

Snieder R and Wapenaar K (2010). Imaging with ambient noise. Phys Today 63(9): 44-49.

Wapenaar K, Draganov D and Robertsson J O A eds (2008). Seismic Interferometry: History and Present Status, Geophysics Reprints Series No. 26. Society of Exploration Geophysicists, doi:10.1190/1.9781560 801924.

Weaver R L (2005). Information from seismic noise. Science 307: 1 568-1 569.

Acknowledgements I thank comments from Bob Nowack and support from the U.S. National Science Foundation (EAR-0838188). 